\title{
STAAR: a randomised controlled trial of electronic adherence monitoring with reminder alarms and feedback to improve clinical outcomes for children with asthma
}

\author{
Robert W Morton, ${ }^{1,2}$ Heather E Elphick, ${ }^{1,2}$ Alan S Rigby, ${ }^{3}$ William J Daw, ${ }^{1,2}$ \\ David A King, ${ }^{1}$ Laurie J Smith, ${ }^{2}$ Mark L Everard ${ }^{4}$
}

\begin{abstract}
- Additional material is published online only. To view please visit the journal online (http://dx.doi.org/10.1136/ thoraxinl-2015-208171).

'Academic Unit of Child Health, University of Sheffield, Sheffield, South Yorkshire, UK 2Department of Respiratory Medicine, Sheffield Children's Hospital, Sheffield, South Yorkshire, UK

${ }^{3}$ Hull York Medical School University of Hull, Hull, Yorkshire, UK

${ }^{4}$ School of Paediatrics and Child Health, Princess

Margaret Hospital University of Western Australia, Perth, Western Australia, Australia
\end{abstract}

\section{Correspondence to} Dr Robert W Morton, Sheffield Children's Hospital, Western Bank, Sheffield, South Yorkshire S10 2TH, UK; rwmorton99@gmail.com

Received 8 December 2015 Revised 28 July 2016 Accepted 6 October 2016 Published Online First 4 November 2016

\begin{abstract}
Background Suboptimal adherence to inhaled steroids is common in children with asthma and is associated with poor disease control, reduced quality of life and even death. Previous studies using feedback of electronically monitored adherence data have demonstrated improved adherence, but have not demonstrated a significant impact on clinical outcomes. The aim of this study was to determine whether introduction of this approach into routine practice would result in improved clinical outcomes.

Methods Children with asthma aged 6-16 years were randomised to the active intervention consisting of electronic adherence monitoring with daily reminder alarms together with feedback in the clinic regarding their inhaled corticosteroid (ICS) use or to the usual care arm with adherence monitoring alone. All children had poorly controlled asthma at baseline, taking ICS and long-acting $\beta$-agonists. Subjects were seen in routine clinics every 3 months for 1 year. The primary outcome was the Asthma Control Questionnaire (ACQ) score. Secondary outcomes included adherence and markers of asthma morbidity.
\end{abstract}

Results 77 of 90 children completed the study (39 interventions, 38 controls). Adherence in the intervention group was $70 \%$ vs $49 \%$ in the control group ( $p \leq 0.001)$. There was no significant difference in the change in $A C Q$, but children in the intervention group required significantly fewer courses of oral steroids $(p=0.008)$ and fewer hospital admissions $(p \leq 0.001)$.

Conclusions The results indicate that electronic adherence monitoring with feedback is likely to be of significant benefit in the routine management of poorly controlled asthmatic subjects.

Trial registration number NCT02451709; pre-result.

\section{INTRODUCTION}

Adherence to inhaled steroids is often suboptimal in children with asthma, resulting in poor disease

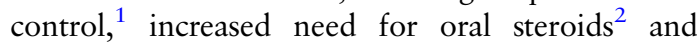
decreased lung function. ${ }^{3}$ This leads to increased healthcare utilisation and associated cost. ${ }^{4}$ The recent National Review of Asthma Deaths in the UK reported that poor adherence was associated with $34 \%$ of deaths due to asthma, emphasising the significance of the problem. ${ }^{5}$

\section{Key messages}

What is the key question?

- Can electronic adherence monitoring with feedback and alarms improve clinical outcomes in children with poorly controlled asthma?

What is the bottom line?

- Electronic monitoring with feedback and alarms improved adherence, decreased hospital admissions and courses of oral steroids required.

\section{Why read on?}

- This study provides the first unequivocal evidence that adherence monitoring with feedback can impact on important clinical outcomes when used in the management of children with poorly controlled asthma.

Large adult population studies and smaller paediatric studies have shown that adherence rates of $75 \%-80 \%$ are required to significantly improve asthma control. ${ }^{6-8}$ However, when objectively measured, the average rate of adherence in children with asthma is around 50\%, some way below this desired therapeutic level. ${ }^{9}$

Subjective and indirect measurements of adherence have been shown to overestimate rates due to patients wanting to be looked upon favourably by their clinician-the social desirability bias. ${ }^{10}{ }^{11}$ Electronic monitoring devices (EMDs) record adherence rates by logging the exact date and time an inhaler is actuated, with modern devices proven to be highly accurate and reliable in the clinical setting. ${ }^{12}$ Monitoring allows intentional adherence barriers such as negative illness perceptions or medication beliefs to be identified, and addressed with regular open dialogue. ${ }^{13-15}$ Reminder alarms built into the devices can address non-intentional practical barriers such as simply forgetting to take the medication. ${ }^{16}$

Due to their cost, the British Thoracic Society (BTS) has questioned the viability of EMDs outside the research setting, ${ }^{17}$ and The National Institute 
for Health and Care Excellence has recommended further studies to investigate their use clinically. ${ }^{18}$

An Australian study demonstrated that children's adherence increased when electronic data were fed back, although it was underpowered to show any improvement in clinical outcomes. ${ }^{19}$ A recent study in New Zealand improved self-reported asthma control in the short-term using electronic monitoring, no feedback and reminder alarms. ${ }^{20}$ However, this study involved seeing subjects out of clinic every 2 months, used covert monitoring and had no effect on objective clinical outcomes.

In order to determine whether clinically relevant benefits could be observed in a routine clinical setting through the use of electronic monitoring, we undertook a study in which children with poorly controlled asthma were randomised to the use of reminder alarms and feedback or routine care. We hypothesised that by addressing both the intentional and non-intentional adherence barriers, rates would increase to a degree necessary to improve asthma control and clinical outcomes.

\section{METHODS}

The STudy of Asthma Adherence Reminders study was a multicentre, open-label, parallel group randomised controlled trial, with an allocation ratio of 1:1. Written consent was taken from the parents/carers of all participants, and ethical approval for the study was granted by the South Yorkshire Research Ethics committee, REC reference 13/YH/0289. The protocol was registered with ClinicalTrials.gov, number NCT02451709.

\section{Participants and setting}

Children with doctor-diagnosed asthma aged 6-16 years attending hospital clinics in Sheffield or Rotherham were screened for eligibility. Participants had to be taking regular inhaled steroids, with no change in their medication in the last month and an Asthma Control Questionnaire (ACQ) score of at least 1.5, indicating they had poorly controlled asthma. ${ }^{21}$ Participants who could not speak English or had another significant chronic condition were excluded. The EMDs available for this trial were only compatible with seretide or symbicort inhalers. Therefore, all participants were at BTS level 3 at the start of the trial.

\section{Interventions}

Prior to randomisation, all participants had their inhaler technique checked by a qualified asthma nurse, and received a brief asthma education session, emphasising the importance of taking inhaled steroids regularly. All participants were reviewed in their routine asthma clinics 3 monthly and all treatment decisions were made by the clinical team. A member of the study team downloaded data from the EMD at each visit.

\section{Intervention group}

Participants in the intervention group had a commercially available EMD attached to their regular inhaler. The 'Smartinhalers' and 'Smartturbos' are manufactured by Adherium (Auckland, New Zealand) (figure 1). They have a CE safety mark and are validated for adherence monitoring in asthma. ${ }^{12}$ Participants were told the devices monitored the date and time of all actuations. At clinic visits, the adherence data from the previous 3 months were uploaded to the website http://www. smartinhalerlive.com, which displays the data graphically. These data were reviewed with the patient and parent/carer (figure 2). Open, non-judgemental discussions were held about the adherence rate, barriers identified and, if necessary, personalised strategies for improvement were devised. Devices were set to play reminder alarms (music or character noises), with different

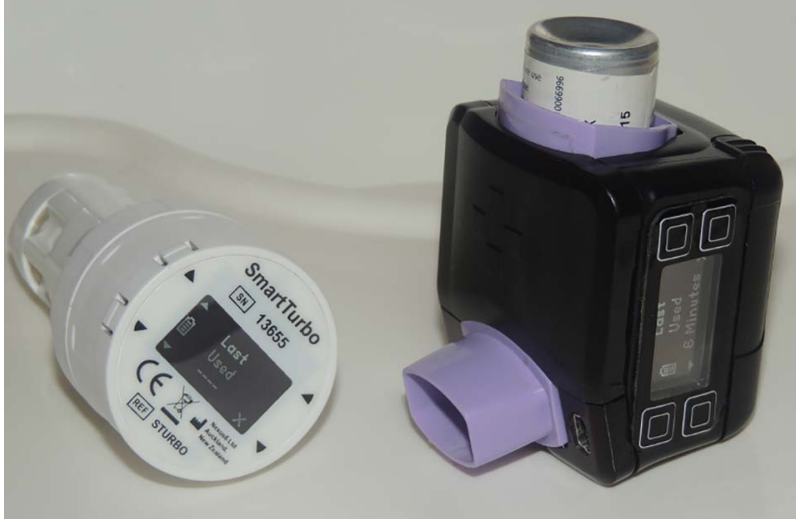

Figure 1 'Smartturbo' and 'Smarttrack' electronic adherence monitors. Adherium, New Zealand.

times agreed for weekdays and weekends. Alarms sounded for $5 \mathrm{~s}$, every minute for $15 \mathrm{~min}$ (or until actuation), if the inhaler had not been actuated within the previous 6 hours of the specified time. The devices were locked to prevent tampering. Times were reviewed each study visit and changed if necessary.

\section{Control group}

Control participants had the same EMDs attached to their regular inhaler, they were also told the devices monitored how much the inhalers were taken, but that these data would not be reviewed. Participants were seen in their standard asthma clinic and the data were downloaded, but not reviewed. The alarms were disabled, and the devices locked.

\section{Primary outcome}

The primary outcome for the study was change in the ACQ score at 3, 6, 9 and 12 months. This questionnaire has been validated for the use in children aged 6-16 years. ${ }^{22}$ It integrates values generated by six clinical questions related to symptoms in the previous week and a value related to $\mathrm{FEV}_{1} \%$ predicted to generate a score of $0-6$. The score is inversely related to asthma control with a high score indicating poor control.

\section{Secondary outcomes}

Secondary outcomes recorded at baseline and each visit were $\mathrm{FEV}_{1} \%,{ }^{23}$ number of unplanned attendances to general practitioner (GP)/emergency department (ED) for asthma since last visit (as reported by parents), number of courses of oral steroids required, number of days off school due to asthma, use of $\beta$-agonists in the past week and BTS level of asthma therapy. Quality of life was measured using the Mini Paediatric Asthma Quality of Life Questionnaire (mini PAQLQ). ${ }^{24}$ The adherence rate was calculated for each 3-month period, both morning and afternoon doses, and recorded as a percentage. This was calculated as number of doses actually taken/number of doses prescribed $\times 100$. The daily adherence was capped at $100 \%$, to avoid falsely increased values due to dose dumping. The overall 3 monthly figure was a mean of each daily \%. Parental beliefs about inhaled steroids and perceptions about asthma were recorded at baseline and 12 months with the Beliefs about Medicines Questionnaire (BMQ) and the Brief Illness Perceptions Questionnaire (IPQ). ${ }^{25} 26$

\section{Sample size}

The sample size was calculated using a repeated measures analysis for four follow-up visits. The Minimal Clinically Important 


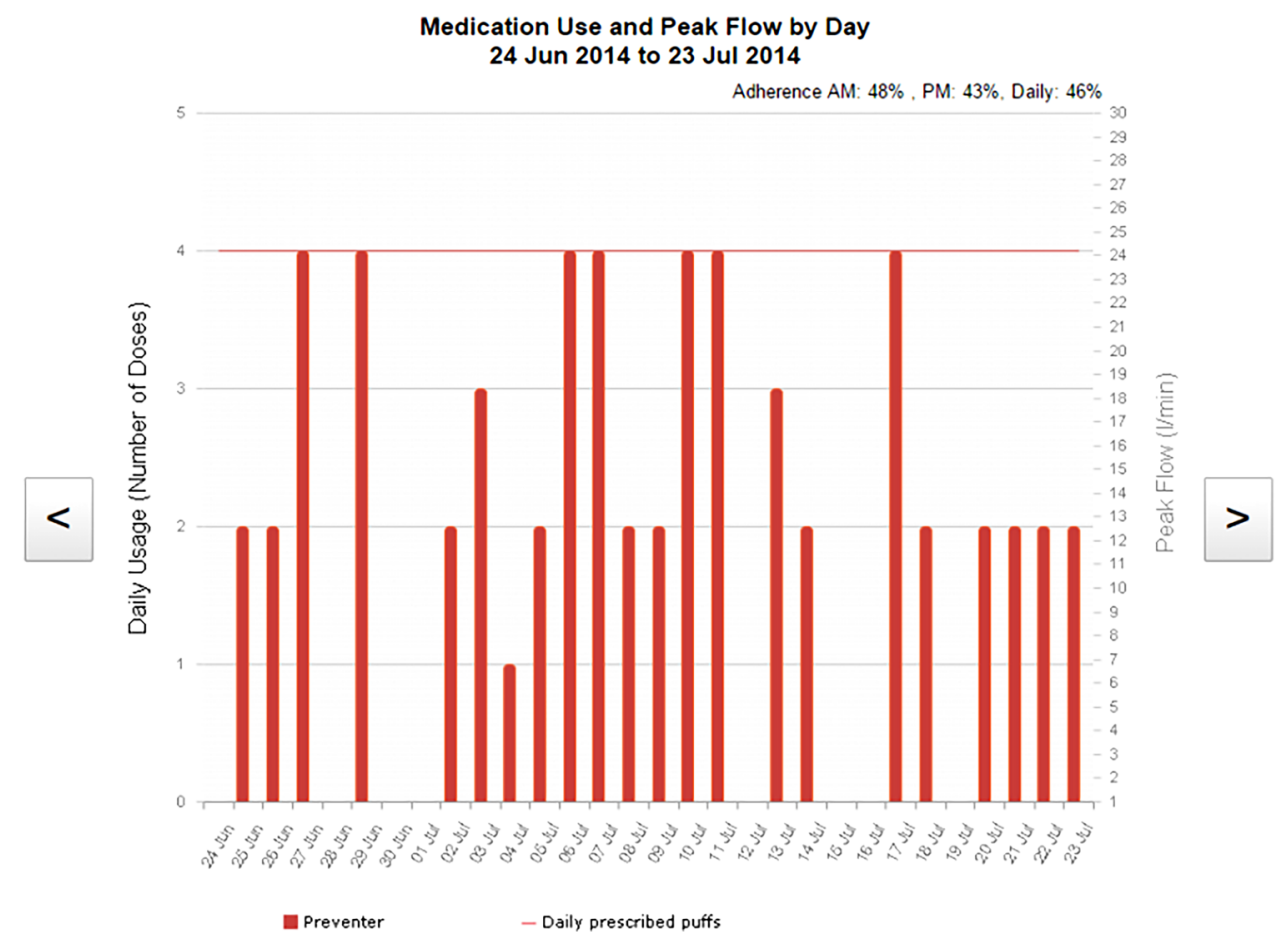

Figure 2 Adherence review graph from http://www.smartinhalerlive.com. The number of daily doses taken is the y axis, with the date on the $x$ axis. In this example, the participant was prescribed four doses on the first date, but only took two doses.

Difference (MID) for the ACQ is $0.5 .^{22}$ Using a repeat measure analysis table, we calculated for a significance of $5 \%(\alpha=0.05)$, and a power of $80 \%$, with a repeated measure correlation of 0.4 , that $\mathrm{n}=76 .{ }^{27}$ To allow for a $15 \%$ attrition rate, we aimed to recruit 90 participants to the study.

\section{Randomisation}

Participants were randomised using permuted block randomisation, with an allocation of $1: 1$ created from a computer generated random number sequence. The allocation of subjects involved phoning the independent holder of the randomisation code.

\section{Blinding}

Due to the nature of the intervention, neither the participants nor the study team were blinded. In the intervention group adherence data were made available to clinicians if requested, but not in the control group.

\section{Statistical analysis}

The ACQ and $\mathrm{FEV}_{1} \%$ were compared statistically by two approaches. First, by calculating a paired difference between 12 months and baseline for each group separately. The 'difference of the difference' between treatments was compared by an independent t-test. A 95\% CI was calculated. Second, an area under the curve (AUC) was calculated for each group and the between-group difference was determined by an independent t-test. The AUC is a mean value weighted by time. ${ }^{28}$ An arbitrary level of 5\% statistical difference was assumed (two-tailed). Incident rates were estimated by Poisson regression, and compared by incident rate ratios (IRRs). The assumption of Poisson regression $($ mean $=$ variance) was confirmed. Continuously distributed data were summarised by the median (quartiles); categorical data by $\mathrm{n}(\%)$. A sensitivity analysis was carried out on the primary outcome measure (ACQ) using multiple imputation.
The approach used is detailed in online supplementary appendix 1, with charts demonstrating the patterns for missing data. The 'Stata' statistical computer package was used for data analysis.

\section{RESULTS}

Ninety children (81 Sheffield, 9 Rotherham) were recruited between October 2013 and August 2014, figure 3 shows the flow diagram of these participants.

\section{Baseline characteristics}

The baseline characteristics are shown in table 1 .

\section{Primary outcome}

The ACQ decreased in both groups between the baseline and the 3 -month visit. It fell by 1.0 in the control group and 0.9 in the intervention group $(p=0.35)$, both significantly exceeding the MID for changes in ACQ scores of $0.5 .^{22}$ This improvement was maintained to 12 months in both treatment arms (figure 4). At 12 months there were no significant differences between the two groups for either the mean change from baseline (table 2) or the AUCs (table 3). The individual participant trajectories for ACQ across the study visits are shown in online supplementary appendix 1 (figure A3).

\section{Secondary outcomes}

Average adherence over the 12 months for the intervention group was $70 \%$ vs $49 \%$ for the control group $(p \leq 0.001)$. Higher mean and median adherence rates were maintained for the 12 -month period in the intervention group, but declined over time in the control group (figure 5). Twenty participants in the intervention group and six in the control group had a mean adherence rate of $>80 \%$ for the 12 -month period. Four participants in the intervention group and 11 in the control group had rates of $<30 \%$. 


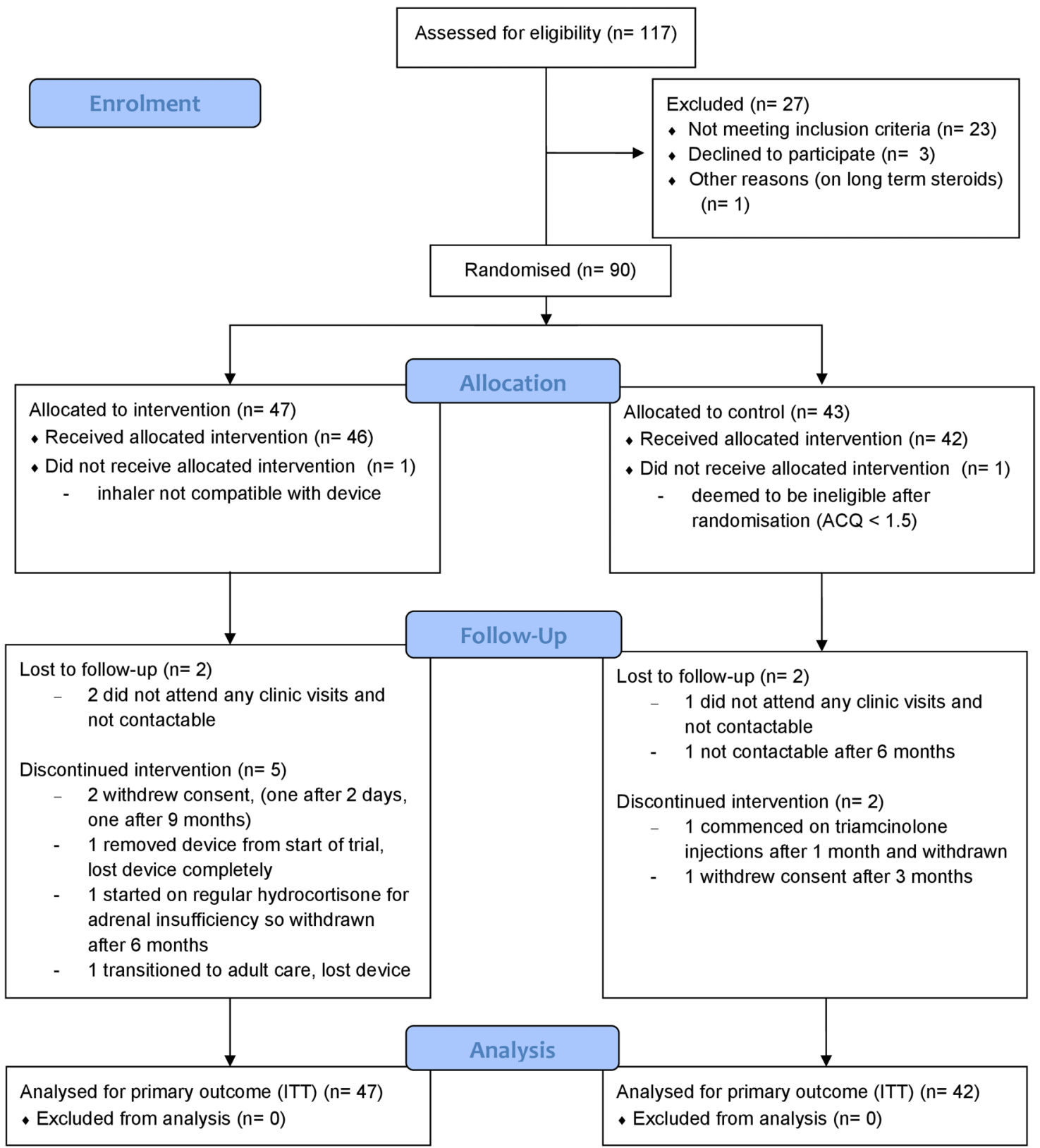

Figure 3 CONSORT 2010 flow diagram showing progress of participants through trial. ACQ, Asthma Control Questionnaire; ITT, intention to treat.

Event rates are shown in table 4 . The mean (SD) days in study was 351 (117) days for the intervention group and 358 (101) days for the control group. Figure 6 shows the timing of rescue oral steroids required during the study.

There were no significant differences between the two groups for short-acting $\beta$-agonist use, change in BTS stage, mini PAQLQ, BMQ or IPQ scores. $\mathrm{FEV}_{1} \%$ improved in both treatment arms, with no significant difference between treatments at 12 months compared with baseline (table 2). The mean (SD) beclomethasone equivalent inhaled corticosteroid dose at the end of the study was $673(303) \mu \mathrm{g}$ in the intervention group and 767 (369) $\mu \mathrm{g}$ in the control group.

\section{Clinic visits}

Patients did not attend (DNA) or cancelled 143 scheduled appointments (73 interventions, 66 controls). Appointments were rescheduled, but where this was not possible, 35 nonclinical study visits (15 interventions, 20 controls) were performed. As a result of these missed study visits, only $41 \%$ of participants in the intervention group received feedback at all three time points (3,6 and 9 months). Missing data for ACQ and adherence are shown in tabulated form in online supplementary appendix 1 .

\section{Issues with electronic adherence monitors}

Table 5 shows the frequency of devices lost, forgotten or broken. Missed data from forgotten devices were downloaded at a later clinic appointment. Reasons given for being damaged (just reported or actually broken) included lost/flat battery, dropped on floor, dropped in liquid, alarms not starting, alarms not stopping, screens peeled off.

\section{DISCUSSION}

The use of electronic adherence monitoring with feedback and regular alarms by children with poorly controlled asthma produced significantly increased adherence rates that were 
Table 1 Baseline data

\begin{tabular}{|c|c|c|}
\hline & $\begin{array}{l}\text { Intervention } \\
\text { group }(n=47)\end{array}$ & $\begin{array}{l}\text { Control group } \\
(n=42)\end{array}$ \\
\hline Age (years) & $10.4(2.9)$ & $10.2(2.9)$ \\
\hline Sex male & $28(60 \%)$ & $22(52 \%)$ \\
\hline ACQ & $2.5(0.8)$ & $2.3(0.7)$ \\
\hline $\mathrm{FEV}_{1} \%$ & $87.2(14.9)$ & $88.0(13.4)$ \\
\hline PQL & $4.3(1.5)$ & $4.6(1.2)$ \\
\hline ICS dose & $697.9(348.6)$ & $664.3(280.1)$ \\
\hline BTS & $3.5(0.6)$ & $3.4(0.5)$ \\
\hline Proportion BTS $\geq 4$ & $51 \%$ & $43 \%$ \\
\hline GP/ED visits & $1.9(2.2)$ & $2.1(2.0)$ \\
\hline$\beta$-Agonist & $2.5(1.3)$ & $2.3(1.3)$ \\
\hline School days missed & $3.5(4.4)$ & $3.8(5.7)$ \\
\hline Oral steroids & $1.2(1.8)$ & $1.2(1.3)$ \\
\hline Hospital admissions & $0.3(0.6)$ & $0.2(0.6)$ \\
\hline BMQ score & $2.5(0.5)$ & $2.6(0.4)$ \\
\hline IPQ score & $5.6(1.3)$ & $5.3(0.9)$ \\
\hline Ethnicity WB & $30(64 \%)$ & $24(57 \%)$ \\
\hline BA & $3(6 \%)$ & $6(14 \%)$ \\
\hline $\mathrm{BP}$ & $11(23 \%)$ & $11(26 \%)$ \\
\hline $\mathrm{BI}$ & $0(0 \%)$ & $1(2 \%)$ \\
\hline $\mathrm{AO}$ & $1(2 \%)$ & $0(\%)$ \\
\hline $\mathrm{BC}$ & $2(4 \%)$ & $0(\%)$ \\
\hline Time from asthma diagnosis (years) & $6.0(3.7)$ & $6.7(3.7)$ \\
\hline \multicolumn{3}{|c|}{$\begin{array}{l}\text { Data are mean (SD) or } n(\%) \text {. } \\
\text { Calculations subject to rounding errors. } \\
\text { GP/ED visits, school days missed, oral steroids required and hospital admissions are } \\
\text { all parent-reported events over the previous } 3 \text { months. } \\
\text { ACQ, Asthma Control Questionnaire; AO, Asian other; BA, black African; BC, black } \\
\text { Caribbean; } \beta \text {-agonist use-score on ACQ question; BI, British Indian; BMQ, Beliefs } \\
\text { about Medicines Questionnaire; BP, British Pakistani; BTS, British Thoracic Society; } \\
\text { FEV }_{1} \% \text {, FEV } \% \text { predicted; ICS dose, inhaled corticosteroid dose (beclomethasone } \\
\text { equivalent); IPQ, Illness Perceptions Questionnaire; PQL, Paediatric Quality of Life } \\
\text { Questionnaire; WB, white British. }\end{array}$} \\
\hline
\end{tabular}

maintained over the 12 months. This improvement was associated with a significant decrease in the number of exacerbations requiring a course of oral steroids or an admission to hospital. This is the first study to our knowledge that has shown that regular alarms and feedback of electronic adherence data have a significant effect on a number of clinically relevant outcomes. Importantly, the intervention was built into routine clinical care and the benefits were sustained over the 12-month study period. Self-reported asthma control improved significantly in both groups, with the change in ACQ being considerably greater than the reported minimally important difference of 0.5 . There was no significant difference in the change in ACQ between the groups.

Previous studies using this approach have also demonstrated improved adherence through the use of reminders with or without feedback, but they have failed to demonstrate a significant difference in clinical outcomes. These studies have variously involved relatively well-controlled and adherent subjects, ${ }^{29}$ relatively mild asthmatic subjects managed in the community $^{30}$ or have been too small to demonstrate significant difference. ${ }^{19}$ In this study, all the subjects had 'poorly controlled asthma' (ACQ $\geq 1.5$ ), and hence there was possibly greater potential to have an impact than those included in previous studies. Our results are consistent with those of Williams et $a{ }^{6}{ }^{6}$ who in a study of 298 adults with asthma found that patients with an adherence in excess of $75 \%$, as assessed by pharmacy records, were significantly less likely to require an ED visit, a course of oral steroids or a hospital admission.

The results from this study suggest that this approach is likely to be beneficial if introduced into routine clinical care, at least in those children with poor asthma control. It is likely that the intervention resulted in an overall reduction in health costs, given that the majority of the direct costs associated with asthma are attributable to hospital admissions, or exacerbations sufficient to require intervention. ${ }^{31}$ In this study, the hospitalisation rate was five times higher in the control group despite self-reported symptoms being similar. This approximates to the prevention of 12 hospitalisations in 1 year among the 47 intervention subjects giving an approximate figure for the number to treat of 3.25. There would appear to be cost saving in using this approach, even at current prices $(£ 120$ per device). The BTS questioned the utility of this approach due to high device cost, but its introduction into routine practice would reduce unit price, as it would drive competition and innovation.
Figure 4 Box and whisker plot showing median Asthma Control Questionnaire (ACQ) scores over time for groups $A$ (intervention) and $B$ (control).

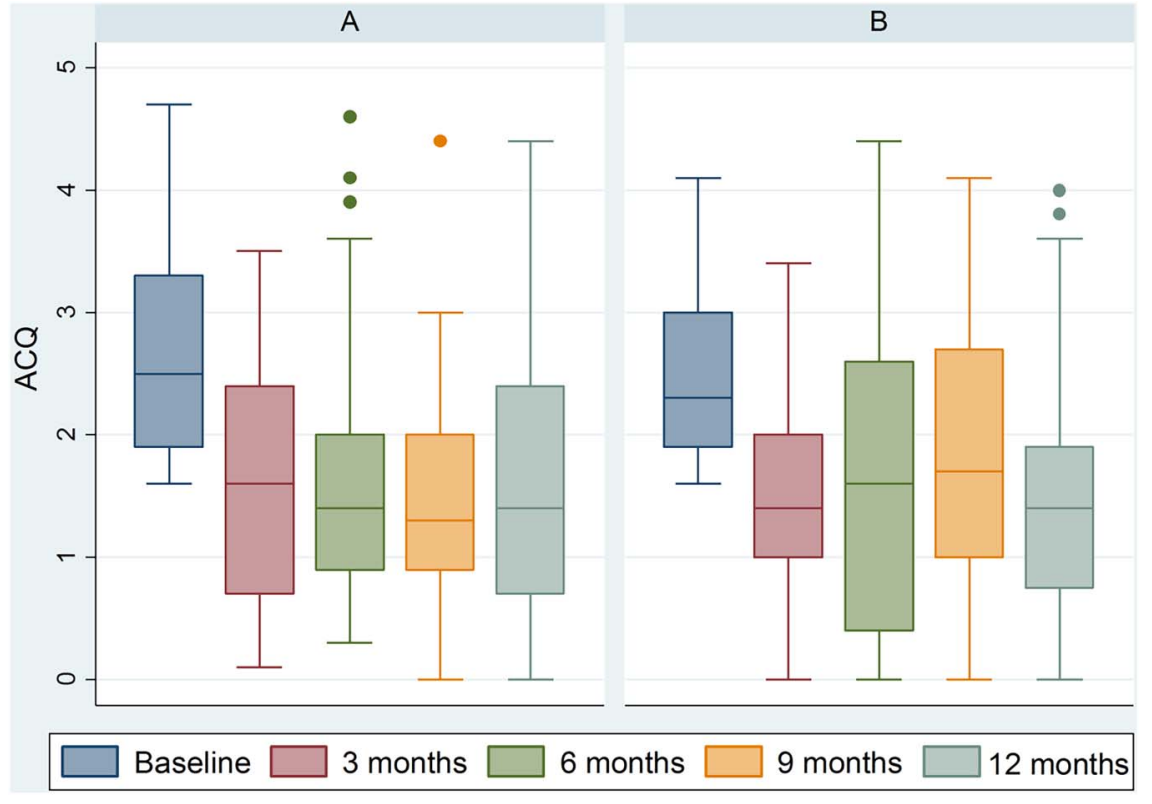


Table 2 Outcome measures for ACQ and $\mathrm{FEV}_{1} \%$

\begin{tabular}{|c|c|c|c|c|c|c|c|c|c|}
\hline Outcome & $\begin{array}{l}\text { Intervention } \\
\text { baseline }\end{array}$ & $\begin{array}{l}\text { Intervention } \\
12 \text { months }\end{array}$ & $\begin{array}{l}\text { Paired mean } \\
\text { difference }\end{array}$ & $\begin{array}{l}\text { Control } \\
\text { baseline }\end{array}$ & $\begin{array}{l}\text { Control } \\
12 \text { months }\end{array}$ & $\begin{array}{l}\text { Paired mean } \\
\text { difference }\end{array}$ & $\begin{array}{l}\text { Difference of } \\
\text { the difference }\end{array}$ & $95 \% \mathrm{Cl}$ & p Value \\
\hline ACQ & $2.65(0.12)$ & $1.58(0.19)$ & $-1.14(0.21)$ & $2.47(0.12)$ & 1.50 (1.07) & $-0.95(0.77)$ & $-0.18(0.28)$ & -0.76 to 0.38 & 0.51 \\
\hline $\mathrm{FEV}_{1} \%$ & $87.23(2.77)$ & $91.37(1.33)$ & $3.00(1.67)$ & $88.00(1.07)$ & $88.97(2.55)$ & $1.54(2.18)$ & 1.45 (3.68) & -4.00 to 6.91 & 0.59 \\
\hline
\end{tabular}

Table 3 Comparison of areas under the curve for ACQ

\begin{tabular}{lcccc}
\hline Intervention & Control & Difference & $95 \% \mathrm{Cl}$ & p Value \\
\hline $1.7(0.13)$ & $1.6(0.14)$ & $0.09(0.18)$ & $(-0.26$ to 0.45$)$ & 0.52 \\
\hline ACQ, Asthma Control Questionnaire.
\end{tabular}

Adherence in the control group fell progressively over the year, consistent with results from previous studies. ${ }^{29} 3032$ At $49 \%$ the median adherence was similar to the value of $53 \%$ derived from our analysis of 18 previous studies using electronic monitors to quantify adherence rates in children. ${ }^{9}$ In contrast, the intervention group maintained a significantly higher level of adherence throughout the study and this was associated with the important improvements in clinical outcomes. While both groups required fewer courses of oral steroids during the study compared with baseline rates, significantly more courses were required in the control group throughout. This difference between groups was more marked at 9 and 12 months, with rates increasing in the control group in the second 6 months (figure 6). Similar patterns of increasing control group rates in the second 6 months were seen for hospital admissions, days off school and GP/ED visits. This would suggest the benefit of this intervention was over a prolonged period of time, maintaining increased adherence rates with regular feedback and discussion.

Which part of the intervention had the greatest impact is unclear. A previous study found reminders alone can have an impact on adherence, but this was in a self-selecting group of well-controlled adult subjects whose mean adherence was high (74\%) in the control group. ${ }^{30}$ It is likely that the feedback in this study played a major role in sustaining improved adherence rates, since other studies using reminders alone have observed declining adherence over time. ${ }^{20}{ }^{29}$ Burgess et $a l^{19}$ also reported sustained improvements in adherence over time through feedback though the study only ran for 4 months and subjects were seen every 4 weeks. Accurate electronic data that could be discussed with the family appeared to facilitate an open and honest discussion about adherence and the barriers encountered, leading to evolving practical solutions. Social desirability could be used in a positive way to influence medication taking behaviour. However, for some participants the interventions did not appear to impact on adherence and there were a disproportionate number in this group who reported damaged or lost devices. This approach may not fully address the issue of intentional non-adherence, but it does help to confirm the problem, should the devices remain operational.

In support of the argument that feedback and discussion are important are the results from a recent study which demonstrated that in 220 children, despite improving self-reported asthma control, electronic monitoring and alarms had no effect on objective clinical outcomes. ${ }^{20}$ Subjects were seen out of clinic every 2 months for 6 months, with adherence declining at each time point in both groups, possibly leading to the initial impact on exacerbations disappearing beyond 2 months. Importantly, the participants received no direct feedback, preventing the opportunity to initiate an open dialogue. Moreover, covert adherence monitoring was used, an approach that cannot be recommended in routine practice.
Figure 5 Box and whisker plot showing median adherence rates over time for groups A (intervention) and B (control).

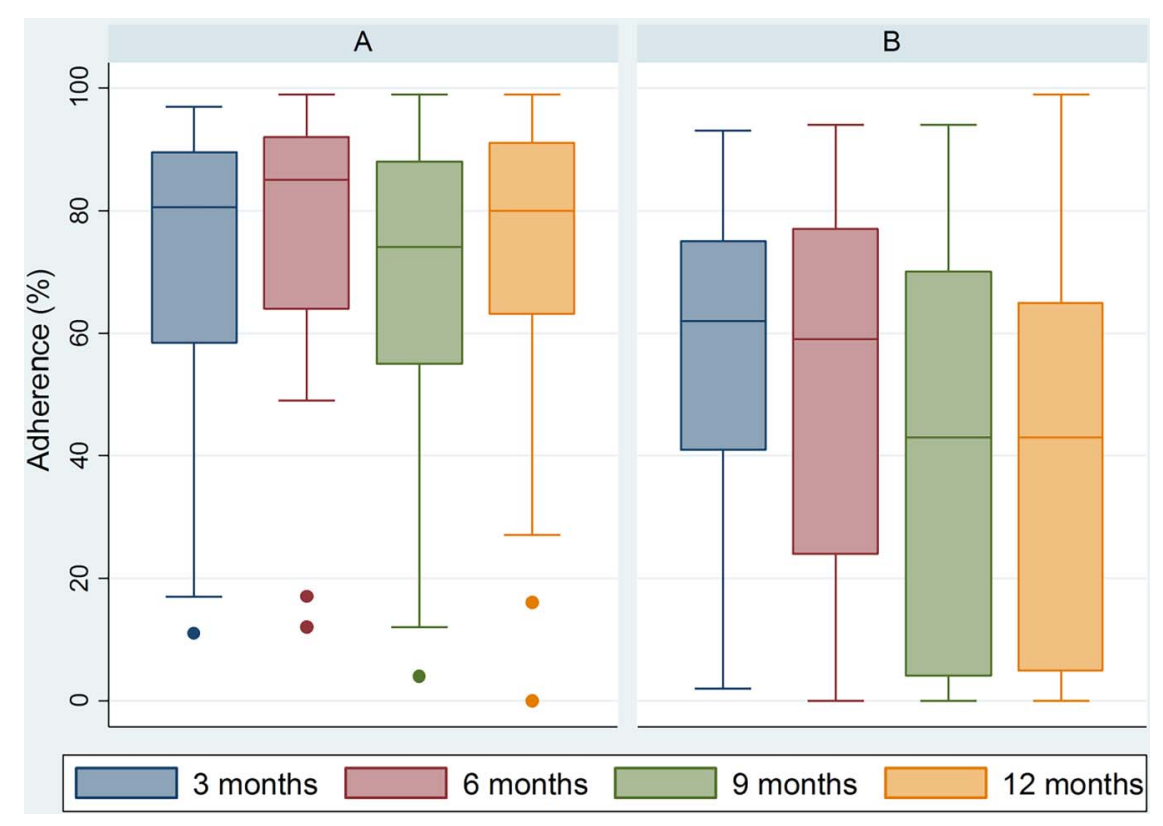

Morton RW, et al. Thorax 2017;72:347-354. doi:10.1136/thoraxjnl-2015-208171 
Table 4 Incident rates for clinical outcomes

\begin{tabular}{|c|c|c|c|c|c|}
\hline & \multicolumn{2}{|c|}{$\begin{array}{l}\text { Event rate (per } 100 \\
\text { child days) }\end{array}$} & \multirow{2}{*}{$\begin{array}{l}p \\
\text { Value }\end{array}$} & \multirow[b]{2}{*}{ IRR } & \multirow[b]{2}{*}{$95 \% \mathrm{Cl}$} \\
\hline & Intervention & Control & & & \\
\hline GP/ED visits $(n=193)$ & 0.582 & 0.650 & 0.316 & 1.15 & $\begin{array}{l}0.83 \text { to } \\
1.63\end{array}$ \\
\hline $\begin{array}{l}\text { Days off school due to } \\
\text { asthma }(n=462)\end{array}$ & 1.365 & 1.606 & 0.1 & 1.16 & $\begin{array}{l}0.97 \text { to } \\
1.39\end{array}$ \\
\hline $\begin{array}{l}\text { Courses of oral steroids } \\
(n=156)\end{array}$ & 0.411 & 0.676 & 0.008 & 1.53 & $\begin{array}{l}1.11 \text { to } \\
2.11\end{array}$ \\
\hline $\begin{array}{l}\text { Hospital admissions } \\
(\mathrm{n}=20)\end{array}$ & 0.0254 & 0.129 & $<0.001$ & 4.38 & $\begin{array}{l}1.46 \text { to } \\
12.13\end{array}$ \\
\hline \multicolumn{6}{|c|}{$\begin{array}{l}n=\text { total number of events in study as reported by parents. } \\
\text { Example interpretation: controls are } 53 \% \text { more likely to be prescribed steroids than } \\
\text { those in the intervention. } \\
\text { ACQ, Asthma Control Questionnaire; ED, emergency department; GP, general } \\
\text { practitioner; IRR, incident rate ratio. }\end{array}$} \\
\hline
\end{tabular}

Out of necessity this was an open-label study. The risk of introducing an inadvertent bias was minimised by ensuring both groups had the same number of clinic visits (136 interventions, 124 controls) and ensuring clinical management was undertaken by the patients' usual clinicians. The control participants were aware that their adherence was being monitored in a clinical trial, and the increased rates seen at 3 months may be due to this. ${ }^{33}$ It is possible that true adherence was lower in the intervention group than that recorded as the devices (and all EMDs currently available) simply recorded actuations with no guarantee the medication was inhaled. Future devices are likely to overcome this problem by detecting inhalation using thermistors or other air flow monitors, an approach used successfully in electronic respiratory rate monitors. ${ }^{34}$

The lack of difference in ACQ in the two groups despite what appear to be significant differences in adherence rates is consistent with results reported in other studies. ${ }^{29} 30$ Similarly recent community studies have reported a disconnect between selfreported symptoms and other clinical outcomes such as exacerbations. $^{30}{ }^{35}$ This is possibly because the ACQ score is a subjective measure. Open, honest dialogue facilitated by adherence discussions may have made children more honest about the severity of their symptoms in the intervention group. In contrast, answers from control subjects may have been more guarded, with denial of symptoms, as is the case in standard clinical

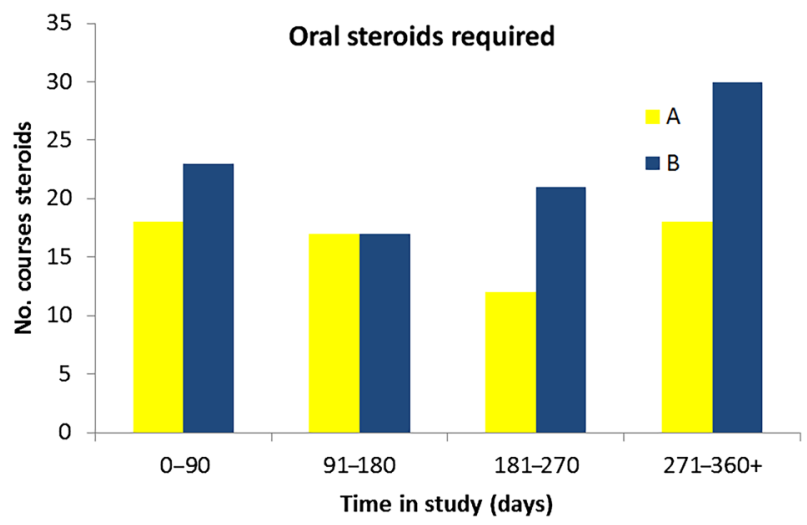

Figure 6 Bar chart demonstrating the number of courses of oral steroids required for different time periods during the study for groups A (intervention) and B (control). Group A total 65 courses, group B total 91 courses.
Table 5 Broken, forgotten and lost devices

\begin{tabular}{lll}
\hline & $\begin{array}{l}\text { Intervention } \\
\text { (47 participants) }\end{array}$ & $\begin{array}{l}\text { Control } \\
\text { (42 participants) }\end{array}$ \\
\hline $\begin{array}{l}\text { Device reported as 'broken' by child } \\
\text { Devices damaged beyond repair (when }\end{array}$ & $23(50 \%)$ & $8(19 \%)$ \\
$\begin{array}{l}\text { inspected by study team, requiring } \\
\text { replacement device) }\end{array}$ & $2(5 \% \%)$ & $18(43 \%)$ \\
$\begin{array}{l}\text { Participant forgot to bring device to } \\
\text { clinic } \\
\text { Device lost completely }\end{array}$ & $10(22 \%)$ & $2(5 \%)$ \\
\hline
\end{tabular}

practice, particularly in adolescents. ${ }^{36}$ Additionally, patients who knew their adherence has been poor may have minimised symptom reporting. ${ }^{13}$ The ACQ scores and $\mathrm{FEV}_{1} \%$ may also reflect short-term influences such as the recent use of oral steroids, which was significantly more common in the control group. The lack of a significant difference in $\mathrm{FEV}_{1} \%$ between groups and poor correlation with exacerbations may be due to $\mathrm{FEV}_{1} \%$ being an inaccurate way of grading asthma severity in children. $^{37}$

This was designed to be a pragmatic study in which asthmatic children continued with usual care with or without the intervention. There was a high rate of participants cancelling appointments, or simply not attending. This appears to reflect the attitude of many patients and their families who do not necessarily always consider asthma a significant condition. The overall DNA rate in this study was 20\% (71/360 appointments, 38 group A, 33 group B), and this compares with a rate of $12 \%$ in respiratory clinics in Sheffield. This may demonstrate a reluctance to attend if the participant felt they may be judged or blamed for poor adherence by the study team or parent. Alternatively, due to their improved clinical condition (shown by improved ACQ), participants may have missed appointments because they felt better, a common reason for non-attendance. ${ }^{38}$ Related to this issue were the high levels of devices reported to be 'broken'. Patel et al reported a Smartinhaler malfunction rate of $1.9 \%$, and $3.5 \%$ lost when assessing 2642 monitors in an adult study. ${ }^{12}$ Our rates of malfunction and loss were much higher (35\% and $8 \%$, respectively), suggesting some of the devices were deliberately broken or lost. The higher rates in the intervention group (50\% broken, $11 \%$ lost) suggest that children are more likely to break or lose their device when their adherence is being openly monitored. The next generation of devices provide automated uploading of data via smartphones such that adherence can be monitored in real-time, which offers significant advantages in terms of logistics and opportunity to influence behaviour. These high rates of damage may suggest that this approach was disliked by the participants. However, the devices were popular and positive feedback was received, with the majority of participants asking to keep their device after the study period. A recent qualitative study to investigate young peoples' opinions on electronic monitoring and feedback using these Smartinhaler devices also reported positive opinions, concluding that this is a popular intervention for both adolescents and their parents. ${ }^{39}$

In summary, these data indicate that significant clinical benefits can be derived from using electronic adherence monitoring with feedback and alarms. A sustained improvement in adherence rates was associated with a decreased number of courses of oral steroids required and hospital admissions when used in a population of poorly controlled asthmatic children using combination therapy. 
Twitter Follow David King at @davidking83

Acknowledgements We would like to thank Drs Sonal Kansra, Kelechi Ugonna, Ruth O'Reilly and Ben Bradbrook for accommodating the study in their asthma clinics. We would like to thank the specialist respiratory nurses Moira Gibbons, Nichola Butler, Anita Critchlow, Emma Sheppard and Bridget Howe for their help in facilitating the study visits in clinic. We would like to thank the respiratory physiologists Karen O'Donnell and Royston Andrew for performing lung function on the participants. We would like to thank our independent pharmacist Jayne Clements for performing the randomisation. Finally, we would like to thank Alexandra Spooner and all the other staff in R\&D at Sheffield Children's Hospital for implementing the study.

Contributors RWM wrote the protocol for the study, applied for and secured ethical approval for the study and applied for and secured funding for the study. He recruited participants, carried out study visits, recorded the results and wrote and edited the manuscript. HEE helped write the protocol, helped apply for funding, supervised the implementation of the study and helped write and edit the manuscript. ASR helped write the protocol, performed all the statistical analysis for the study and helped write and edit the manuscript. WJD carried out study visits and helped edit the manuscript. DAK helped apply for ethical approval, helped apply for funding, recruited participants, carried out study visits and helped edit the manuscript. LJS recorded and analysed the lung function for all the participants in the study and helped edit the manuscript. MLE devised the concept of the study, wrote the protocol and helped write and edit the manuscript.

Funding This study was funded by the Sheffield Children's Hospital Charity.

Competing interests None declared.

Ethics approval South Yorkshire Research Ethics Committee.

Provenance and peer review Not commissioned; externally peer reviewed.

\section{REFERENCES}

1 Jentzsch NS, Camargos P, Sarinho ES, et al. Adherence rate to beclomethasone dipropionate and the level of asthma control. Respir Med 2012;106:338-43.

2 Bender B, Zhang L. Negative affect, medication adherence, and asthma control in children. J Allergy Clin Immunol 2008;122:490-5.

3 Duncan $\mathrm{CL}$, Hogan MB, Tien KJ, et al. Efficacy of a parent-youth teamwork intervention to promote adherence in pediatric asthma. J Pediatr Psychol 2013:38:617-28

4 McGrady ME, Hommel KA. Medication adherence and health care utilization in pediatric chronic illness: a systematic review. Pediatrics 2013;132:730-40.

5 Royal_College_of_Physicians. Why asthma still kills: the National Review of Asthma Deaths (NRAD) Confidential Enquiry Report. London: RCP, 2014

6 Williams LK, Peterson EL, Wells K, et al. Quantifying the proportion of severe asthma exacerbations attributable to inhaled corticosteroid nonadherence. J Allergy Clin Immunol 2011;128:1185-1191.e2.

7 Klok T, Kaptein AA, Duiverman EJ, et al. It's the adherence, stupid (that determines asthma control in preschool children)! Eur Respir J 2014;43:783-91.

8 Lasmar L, Camargos P, Champs NS, et al. Adherence rate to inhaled corticosteroids and their impact on asthma control. Allergy 2009;64:784-9.

9 Morton RW, Everard ML, Elphick HE. Adherence in childhood asthma: the elephant in the room. Arch Dis Child 2014;99:949-53.

10 Bender B, Wamboldt FS, O'Connor SL, et al. Measurement of children's asthma medication adherence by self report, mother report, canister weight, and Doser CT. Ann Allergy Asthma Immunol 2000;85:416-21.

11 Jentzsch NS, Camargos PA, Colosimo EA, et al. Monitoring adherence to beclomethasone in asthmatic children and adolescents through four different methods. Allergy 2009;64:1458-62.

12 Patel M, Pilcher J, Travers J, et al. Use of metered-dose inhaler electronic monitoring in a real-world asthma randomized controlled trial. J Allergy Clin Immunol Pract 2013;1:83-91.

13 Horne R, Weinman J. Self-regulation and self-management in asthma: exploring the role of illness perceptions and treatment beliefs in explaining non-adherence to preventer medication. Psychol Health 2002;17:17-32

14 Klok T, Kaptein AA, Duiverman EJ, et al. High inhaled corticosteroids adherence in childhood asthma: the role of medication beliefs. Eur Respir J 2012;40:1149-55.
15 Everard ML. The Emperor's New Clothes II--time for regulators to wake up and take responsibility for unnecessary asthma morbidity: time for the second aerosol 'transition'. Thorax 2013;68:891-3.

16 Burgess SW, Sly PD, Morawska A, et al. Assessing adherence and factors associated with adherence in young children with asthma. Respirology 2008;13:559-63.

17 National Institute for Health and Care Excellence. Asthma: diagnosis and monitoring of asthma in adults, children and young people [Draft for Consultation]. London: NICE, Jan 2015. https://www.nice.org.uk/guidance/gid-cgwave0640/resources/ asthma-diagnosis-and-monitoring-draft-nice-guideline2

18 British Thoracic Society/Scottish Intercollegiate Guidelines Network. BTS/SIGN British guideline on the management of asthma. London: British Thoracic Society, 2014.

19 Burgess SW, Sly PD, Devadason SG. Providing feedback on adherence increases use of preventive medication by asthmatic children. J Asthma 2010;47:198-201.

20 Chan AH, Stewart AW, Harrison J, et al. The effect of an electronic monitoring device with audiovisual reminder function on adherence to inhaled corticosteroids and school attendance in children with asthma: a randomised controlled trial. Lancet Respir Med 2015;3:210-19.

21 Juniper EF, Bousquet J, Abetz L, et al. Identifying 'well-controlled' and 'not well-controlled' asthma using the Asthma Control Questionnaire. Respir Med 2006;100:616-21.

22 Juniper EF, Gruffydd-Jones K, Ward S, et al. Asthma Control Questionnaire in children: validation, measurement properties, interpretation. Eur Respir J 2010:36:1410-16.

23 Quanjer PH, Stanojevic S, Cole TJ, et al. Multi-ethnic reference values for spirometry for the 3-95-yr age range: the global lung function 2012 equations. Eur Respir J 2012;40:1324-43

24 Wing A, Upton J, Svensson $\mathrm{K}$, et al. The standardized and mini versions of the PAQLQ are valid, reliable, and responsive measurement tools. J Clin Epidemiol 2012;65:643-50

25 Broadbent E, Petrie KJ, Main J, et al. The brief illness perception questionnaire. J Psychosom Res 2006;60:631-7.

26 Horne R, Weinman J, Hankins $M$. The beliefs about medicines questionnaire: the development and evaluation of a new method for assessing the cognitive representation of medication. Psychol Health 1999:14:1-24.

27 Frison L, Pocock SJ. Repeated measures in clinical trials: analysis using mean summary statistics and its implications for design. Stat Med 1992;11:1685-704.

28 Matthews JN, Altman DG, Campbell MJ, et al. Analysis of serial measurements in medical research. BMJ 1990;300:230-5.

29 Charles T, Quinn D, Weatherall $M$, et al. An audiovisual reminder function improves adherence with inhaled corticosteroid therapy in asthma. J Allergy Clin Immunol 2007:119:811-16.

30 Foster JM, Usherwood T, Smith L, et al. Inhaler reminders improve adherence with controller treatment in primary care patients with asthma. J Allergy Clin Immunol 2014;134:1260-1268.e3.

31 Bahadori K, Doyle-Waters MM, Marra C, et al. Economic burden of asthma: a systematic review. BMC Pulm Med 2009;9:24.

32 Nikander $\mathrm{K}$, Turpeinen M, Pelkonen AS, et al. True adherence with the Turbuhale in young children with asthma. Arch Dis Child 2011;96:168-73.

33 McCarney R, Warner J, lliffe S, et al. The Hawthorne Effect: a randomised, controlled trial. BMC Med Res Methodol 2007;7:30.

34 Al-Khalidi FQ, Saatchi R, Burke D, et al. Respiration rate monitoring methods: a review. Pediatr Pulmonol 2011:46:523-9.

35 Turpeinen M, Pelkonen AS, Selroos 0 , et al. Continuous versus intermittent inhaled corticosteroid (budesonide) for mild persistent asthma in children-not too much, not too little. Thorax 2012;67:100-2.

36 Osman LM. Psychological factors in asthma control and attack risk. Thorax 2002;57:190-1

37 Paull K, Covar R, Jain N, et al. Do NHLBI lung function criteria apply to children? A cross-sectional evaluation of childhood asthma at National Jewish Medical and Research Center, 1999-2002. Pediatr Pulmonol 2005:39:311-17.

38 Murdock A, Rodgers $C$, Lindsay $H$, et al. Why do patients not keep their appointments? Prospective study in a gastroenterology outpatient clinic. J $R$ Soc Med 2002:95:284-6.

39 Howard S, Lang A, Youle C. Exploring the attitudes of adolescents with asthma towards monitoring and sharing of data on their inhaler use. Eur Respir J 2015;46 (Suppl 59):OA4773. 